hyperbolic infinitesimal transformations. Every non-elliptic transformation in $G_{m n}$ can be generated from an infinite number of distinct infinitesimal transformations; for every such transformation belongs to an infinite number of distinct subgroups.

THeOREM 8. Every finite transformation of the group $G_{m n}$ can be generated by the repetition of an infinitesimal transformation of the group. Every elliptic (non-elliptic) transformation in $G_{m n}$ can be generated from two (an infinite number of) distinct transformations. Hyperbolic transformations for which $k$ is negative can not be generated by either hyperbolic infinitesimal transformation.

UNIVERSITY OF KANSAS, February, 1903.

\title{
STUDY'S GEOMETRY OF DYNAMES.*
}

Geometrie der Dynamen. Die Zusammensetzung von Kräften und verwandte Gegenstände der Geometrie. Von E. STudy. Leipzig, Teubner, 1903. 8mo., 603 pp., 46 figs.

The original purpose of Professor Study's book was to present a systematic geometric treatment of the composition of forces acting on a rigid body, but as the work progressed the idea broadened, resulting in an elaborate treatise on a whole field of geometry, hitherto nearly unknown.

Probably no other work on geometry that has appeared since the memoirs by Klein and by Lie in the early volumes of the Mathematische Annalen contains so many original and fruitful ideas as that under review.

The book is divided into three parts : the first (pages 1-122) treats of the composition of forces as a problem in pure geometry; the second (pages 123-225) treats the same problem analytically, making free use of symbolic notation and of line geometry; the third (pages 226-556) is devoted mainly to the discussion of a transformation first met with in the second part.

The first two parts contain a new geometric theory of the composition of forces and of infinitesimal motions. In the first part no use is made of other branches of mathematics than elementary geometry and trigonometry, but in order to express

* Dyname is the form used by Plücker in his English papers in the London Transactions Roy. Soc. 
himself explicitly the author feels the necessity of introducing over a hundred new terms, which he defines. Apart from the use of so many new words, the matter is presented in a particularly attractive manner. The ideas of Wiener and of Clifford are systematized and generalized. The premises in each case are sharply defined and the theorems are so well stated that the exact meaning is easily grasped.

In order to express a motion as a line transformation, collineation and duality in a linear complex are first treated, then a new transformation is defined as follows: Let a line $x$ be transformed by a general screw motion $S$ into $x^{\prime}$; this is indicated by

$$
x\left(S^{\prime}\right) x^{\prime} .
$$

Associated with every pair of lines $x, x^{\prime}$ is another line $x_{1}$, namely, the bisector of the acute angle and of the common perpendicular of $x, x^{\prime}$. If the lines $x, x_{1}, x^{\prime}$ be connected as follows :

then

$$
x\left(\tau_{1}\right) x_{1}\left(\tau_{2}\right) x^{\prime},
$$

$$
\tau_{1} \tau_{2}=S=\tau_{2} \tau_{1} .
$$

The operation $\tau$ is not a point transformation nor even a contact transformation, for lines which intersect go over into lines skew to each other. The operations of homography and duality can be expressed as linear transformations of line coördinates $p_{i k}$. By introducing a new number it was found that the operation $\tau$ could be expressed as a linear transformation of the $p_{i k}$. The problem then naturally presented itself to determine the meaning of the general linear transformation in the new coordinates.

The third part of the work, comprising about two thirds of the volume, is devoted to the discussion of this problem. In this part is derived a new metrical line geometry which in many respects differs widely from that of Plücker. Although no hint is given the reader regarding the origin of the new idea, it seems to the reviewer to be a sort of generalization of a paper by Klein,* and by the aid of a paper by E. Müller $\dagger$ to have a natural interpretation as follows : Given any conic

\footnotetext{
* "Ein Uebertragungsprinzip und der Pascalsche Satz," Math. Annalen, vol. 22 .

† "Ein Uebertragungsprinzip des Herrn E. Study," Archiv der Mathematik und Physik, series 3, vol. 5, pp. 104-118.
} 


$$
x=\phi_{2}(\lambda), \quad y=f_{2}(\lambda), \quad t=\psi_{2}(\lambda)
$$

in the plane $z=0$. A straight line $a x+b y+c t=0$ will cut the conic in two points $\lambda_{1}, \lambda_{2}$. Let the (real or imaginary) values of $\lambda$ be projected stereographically from the $\lambda$ plane (or plane $z=0$ ) on a sphere. The two points $\lambda_{1}, \lambda_{2}$ will always project into real points on the sphere, and therefore uniquely determine the real line joining them. In this way the $\infty^{4}$ real and imaginary lines of the plane $z=0$ are put in $(1,1)$ correspondence with the $\infty^{4}$ real secants of a sphere. The fundamental elements of the latter are the $\infty^{2}$ tangents, since all the tangents of a pencil are images of a single tangent to the conic. Lines which are conjugate with respect to the conic go over into lines of space which intersect and which are conjugate with regard to the sphere. If the conic and the sphere each be taken as absolute in its respective space the theorem becomes:

Perpendicular lines in the plane go over into intersecting perpendicular lines of space.

The position of a line in the plane is defined by the (real or imaginary) coördinates $a, b, c$. If these numbers be transformed, 16 real parameters are involved, defining a group $G_{16}$. Perpendicularity (the property of being conjugate with regard to the fundamental conic) is preserved by this transformation. Thus far the image lines in space are only the real secants of a sphere; in order to include all the lines of space the radius of the sphere must be infinitely large. The fundamental points are now in the plane at infinity. In order to distinguish between the two points $\lambda_{i}$ on the surface of the sphere and insure that the line connecting them does not lie entirely at infinity, it is convenient to think of all the lines of space being double, or arranged in two superposed sheets. The transformations will now also include similarity, making a $G_{17}$.

Among the more important invariants of this group, which can be established by the preceding considerations, the author mentions the following: (1) parallelism of two lines of the same sheet; (2) a finite line and its perpendicular infinite line ; (3) skew orthogonality and (4) orthogonal intersection of finite lines of different sheets ; (5) intersection of a finite line and an infinite line when belonging to different sheets; (6) orthogonal intersection of two infinite lines of different sheets; (7) three orthogonal transversals of a given line. 
The development here traced is not mentioned by Professor Study, and perhaps he made no use of it; but it furnishes an easy way to understand the subsequent treatment.

In order to obtain the analytic expressions for the transformations of $G_{17}$, we shall reproduce a portion of the paper of Professor Müller. Let

$$
K \equiv \Sigma a_{i k} p_{l m}=0
$$

be the equation of a linear complex. The direction vectors of its axis are $a_{12}: a_{13}: a_{14}$. If the invariant $A=\Sigma a_{i k} a_{l m}$ vanishes, the complex becomes special. In any case the coefficients $a_{i k}$ may be called the coördinates of $K$.

If $K_{1}, K_{2}$ be two special complexes, such that

$$
K=\lambda_{1} K_{1}+\lambda_{2} K_{2}
$$

then the axes of $K_{1}, K_{2}$ are conjugate polars with regard to every complex $K$ of the pencil. In particular, consider

$$
\begin{aligned}
& K_{1}=a_{12} p_{34}+a_{13} p_{42}+a_{14} p_{23}=0 \\
& K_{2}=a_{34} p_{12}+a_{42} p_{13}+a_{23} p_{14}=0
\end{aligned}
$$

The former is coaxial with $K$, the latter is a parallel complex. The complex

$$
K^{\prime}=a_{12} p_{12}+a_{13} p_{13}+a_{14} p_{14}=0
$$

is special; its axis is in the plane at infinity, perpendicular to the axis of $K$. If the special complex whose axis is the conjugate polar of this infinite line with regard to $K$ be $K^{\prime \prime}$, then

$$
K=\mu K^{\prime \prime}+\nu K^{\prime}
$$

Hence $K+\lambda K^{\prime}=\mu K^{\prime \prime}+(\nu+\lambda) K^{\prime}$ always represents a line complex coaxial with $K$.

Consider the transformation with vanishing determinant

$p_{14}=0, \quad p_{4^{2}}=0, \quad p_{34}=0, \quad p_{12}=p_{34}^{\prime}, \quad p_{13}=p_{42}^{\prime}, \quad p_{23}=p_{34}^{\prime}$.

By means of this transformation any line through the origin, as 
axis of a special complex, is transformed into a line at infinity perpendicular to it, and every line in the plane at infinity vanishes identically.

Any general complex $K$ is transformed into a special complex whose axis is perpendicular to the axis of $K$ and lies in the plane at infinity.

The transformation is designated by $\epsilon$, so that

hence

$$
K \epsilon=\epsilon K=K^{\prime}, \quad \epsilon \epsilon K=\epsilon^{2} K=0,
$$

$$
\epsilon^{2}=0 \text {. }
$$

With this further restriction, $\epsilon^{2}=0$, numbers of the form $m+m^{\prime} \epsilon$ obey the ordinary laws of combination of complex numbers except that when $m=0$, the number can not be used as a divisor. Numbers of this character are called dual numbers. When a complex $K$ is multiplied by a dual number it goes over into a coaxial complex.

It is now possible to establish a correspondence between the lines of a sheaf whose vertex is the origin and the plane field of lines in the plane at infinity.

The coördinates $x_{1}, x_{2}, x_{3}$ of a line in a sheaf (or a plane) are defined as dual numbers of the form

$$
x_{1}=a_{12}+\epsilon a_{34}, \cdots
$$

and the $a_{i k}$ are regarded as coördinates of a complex. By multiplying a linear equation by a dual number of the form

$$
a_{14}^{2}+a_{42}^{2}+a_{34}^{2}-\frac{1}{2} A(a) \epsilon,
$$

a special coaxial complex is obtained; hence it is no restriction to regard the $a_{i k}$ as line coördinates.

In the finite part of space this geometry is now identical with Plücker's line geometry, but configurations at infinity are interpreted in an entirely different way. The various preëxistent ideas are skillfully brought into use in a strikingly elegant manner. One unconsciously gets the idea that the work of Hamilton, Clifford, Klein, Segre, Hankel, and others was conveniently designed for this particular purpose. Indeed a large part of the whole field of mathematics is shown to be a sort of stepping stone to line geometry. The admiration of the reader is dampened, 
however, by an unfortunate self-conscious style that fails to do justice to the beauty of the subject. The mode of expression is frequently so involved that one not particularly sympathetic with the author's purpose will find the text very difficult reading. In this respect the third part of the book stands in striking contrast to the first part.

So much as to the method-a method that gives fair promise of very fruitful results. Now as to the applications which the author himself has made of it. The uses of the dual coördinates furnish the subject matter of about two thirds the entire volume. They are identical with Plücker's complex coördinates in the finite part of the plane, but are illusory for configurations at infinity. In order to represent a definite continuum, the author introduces new coördinates, which are called coördinates of the second kind, of the first natural system. They are defined by the equations

$$
\begin{gathered}
a_{i}=a_{4 i} \quad(i=1,2,3) \\
a_{11}=\left|\begin{array}{ll}
a_{42} & a_{31} \\
a_{43} & a_{12}
\end{array}\right|, \quad a_{22}=\left|\begin{array}{ll}
a_{43} & a_{12} \\
a_{41} & a_{23}
\end{array}\right|, \quad a_{33}=\left|\begin{array}{cc}
a_{41} & a_{23} \\
a_{42} & a_{31}
\end{array}\right|,
\end{gathered}
$$

so that

$$
a_{1} a_{11}+a_{2} a_{22}+a_{3} a_{33} \equiv 0 \text {. }
$$

These coördinates are homogeneous in the sense that when $a_{i}$ is replaced by $\rho a_{i}, a_{i i}$ by $\rho^{2} a_{i i}$ the elements remain unchanged.

Finally a line at infinity is replaced by its pole with regard to the absolute circle in that plane. With these coördinates the line space forms a continuum in the same sense as projective geometry does, $i$. e., by giving an added definition to infinite elements. Finally a third system of nine coördinates is defined by the equations

$$
a_{i k l}=a_{i} \cdot a_{k k k}
$$

which with the $a_{i}$ make a system of twelve, between which several identities exist. These new coördinates are used to study configurations of limiting rays in the plane at infinity.

After these preliminary ideas the first application is a detailed and exhaustive classification of linear systems of complexes and the determination of their equations in the simplest form. Joined to this is a correspondingly full discussion of the surface 
formed by the axes in a linear pencil of complexes, namely, the cylindroid and its various degenerations in particular cases. The congruence of axes in a linear sheaf of complexes receives too little attention compared with the length of treatment devoted to related subjects.

An equation of the second degree between the dual numbers $x$ defines a congruence, since the real part (scalar) and the coefficient of $\epsilon$ (vector) must be separately equated to zero. Since the numbers $x$, when interpreted as coördinates in a sheaf, define a quadric cone, these congruences are called conical congruences.

'The discussion of these congruences including the reduction of their equations to canonical forms is the subject matter of the interesting doctor dissertation of Dr. A. B. Pierce.* The paper of Dr. Pierce makes the various transformations of the $G_{17}$ much easier to understand. Pages 1-19 of this paper are concerned with the ordinary dual coördinates, the canonical forms of the equations are given for all cases including the degenerate ones; pages 20-30 are devoted to a similar discussion of the coördinates of the second kind. The remainder of the paper treats of geometric interpretations.

An extensive discussion is given by Study of the congruence determined by

$$
\begin{gathered}
a_{41}=\sigma_{1}, \quad a_{42}=\sigma_{2}, \quad a_{43}=\sigma_{3}, \\
a_{23}=\left(p-e_{1}\right) \sigma_{1}, \quad a_{31}=\left(p-e_{2}\right) \sigma_{2}, \quad a_{: 2}=\left(p-e_{3}\right) \sigma_{3},
\end{gathered}
$$

and the linear sheaves of complexes obtained by varying $p$. The treatment is here projective; the author makes no use of his new coördinates. One can read this chapter (pages 461534) without any knowledge of the preceding portion, although a fair knowledge of line geometry is presupposed. If the parameter $p$ be unrestricted, the $a_{i k}$ are Plücker complex coördinates, but if $A=0$, the $a_{i k}$ are tetrahedral line coördinates. First the case is considered in which

$$
e_{1}>e_{2}>e_{3}, \quad \Sigma e_{i}=0 .
$$

This configuration has been investigated by other writers. $\dagger$

* "Classification and properties of dual conical congruences," Zürich, Meyer and Hendess, 1903. 8vo., 60 pp.

† See Ball's Theory of Screws, 2d edition, p. 170ff, W. Waelsch, "Transversalencongruenzen," Wiener Berichte, 1887, Part I. W. Stahl, " Das Strahlensystem dritter Ordnung und zweiter Classe," Crelle's Journal, vol. 91, where other references are given. 
The congruence is of order 3 and class 2 , dual to the congruence of axes in a linear sheaf of complexes. The discussion of the distribution of the lines on $\infty^{1}$ coaxial hyperboloids and their association to the conjugate congruences $(i$. e., such that every complex of the first sheaf is in involution with all those of the second) is followed by a more thorough study of the parametric hyperboloids themselves. The pedal surface is coincident with the mean surface; it is a particular form of the Steiner surface. The focal surface is the same for both congruences; it is of order 6 and class 4 . This surface is exhaustively discussed, first geometrically, then by means of elliptic functions.

After the treatment of the general case is completed, each particular case is fully discussed. In these 70 pages is found not only a systematic and far-reaching discussion of the configuration named, but also a large number of other problems suggested that might furnish themes for further investigation. The treatment differs essentially from that of other authors in the emphasis put upon particular and degenerate forms. While others confine their attention to the so-called "general case" - a case which in many applications does not exist at all the present work concerns itself much more with each particular type, so that in the applications no exceptions are presented.

In conclusion we would say that the author has produced a work that will for some time furnish material for study and reflection. The style is not inviting, and the treatment is frequently involved, but the author has presented a new field of mathematics. Many commentaries will be necessary to make the work generally accessible, but they will surely come.

Corneil University,

VIRGIL SNYDER. October 12, 1903 .

\section{ENCYKLOPÄDIE DER ELEMENTAR-MATHE- MATIK.}

Ein Handbuch für Lehrer und Studierende. Von HeInRICH Weber und Josef Wellstein. Erster Band. Elementare Algebra und Analysis. Von H. Weber. Leipzig, Teubner, 1903. xiv $+447 \mathrm{pp}$.

IT was with much interest that scholars learned, a few years ago, of the proposed appearance of an Encyklopädie der Mathe- 